\title{
Characterisation of Dermanyssus gallinae glutathione $S$-transferases and their potential as acaricide detoxification proteins
}

\author{
Kathryn Bartley*, Harry W. Wright, Robert S. Bull, John F. Huntley and Alasdair J. Nisbet
}

\begin{abstract}
Background: Glutathione S-transferases (GSTs) facilitate detoxification of drugs by catalysing the conjugation of the reduced glutathione (GSH) to electrophilic xenobiotic substrates and therefore have a function in multi-drug resistance. As a result, knowledge of GSTs can inform both drug resistance in, and novel interventions for, the control of endo- and ectoparasite species. Acaricide resistance and the need for novel control methods are both pressing needs for Dermanyssus gallinae, a highly economically important haematophagous ectoparasite of poultry.

Methods: A transcriptomic database representing D. gallinae was examined and 11 contig sequences were identified with GST BlastX identities. The transcripts represented by 3 contigs, designated Deg-GST-1, -2 and -3 , were fully sequenced and further characterized by phylogenetic analysis. Recombinant versions of Deg-GST-1, -2 and -3 (rDeg-GST) were enzymically active and acaricide-binding properties of the rDeg-GSTs were established by evaluating the ability of selected acaricides to inhibit the enzymatic activity of rDeg-GSTs.
\end{abstract}

Results: 6 of the identified GSTs belonged to the mu class, followed by 3 kappa, 1 omega and 1 delta class molecules. Deg-GST-1 and -3 clearly partitioned with orthologous mu class GSTs and Deg-GST-2 partitioned with delta class GSTs. Phoxim, permethrin and abamectin significantly inhibited rDeg-GST-1 activity by 56, 35 and $17 \%$ respectively. Phoxim also inhibited rDeg-2-GST (14.8\%) and rDeg-GST-3 (20.6 \%) activities.

Conclusions: Deg-GSTs may have important roles in the detoxification of pesticides and, with the increased occurrence of acaricide resistance in this species worldwide, Deg-GSTs are attractive targets for novel interventions.

Keywords: Dermanyssus gallinae, Poultry red mite, Glutathione S-transferase, Acaricide resistance

\section{Background}

The haematophagous poultry red mite [Dermanyssus gallinae (De Geer, 1778)] is the most economically important parasite affecting commercial egg production facilities worldwide, causing an estimated annual production loss of $€ 130$ million in Europe alone [1, 2]. Infestation levels are estimated to range from a "normal" 50,000 mites per bird to over 500,000 mites per bird in severe infestations [3]. The impact on bird health and behaviour can be severe and manifests as agitation and pecking, weight loss and anaemia; with production losses attributed to increase bird mortality, a decrease in egg quality and output, higher feed-conversion rate and mite

\footnotetext{
* Correspondence: Kathryn.Bartley@moredun.ac.uk

Moredun Research Institute, International Research Centre, Pentlands Science Park, Bush Loan, Penicuik, Midlothian EH26 OPZ, UK
}

control costs $[2,4,5]$. In addition, $D$. gallinae is as a potential vector for a number of diseases, some of which may also impact on human health; for example, Erisipelas and Salmonella spp. [6-11].

Chemical-based mite control is the usual approach employed in commercial premises and several organophosphate, pyrethroid, spinosyn and carbamate acaricides are licensed for use in the EU [5]. Resistance to organophosphates, pyrethroids, carbamates and dichlorodiphenyltrichloroethane (DDT) has been identified in red mite populations across the EU [12-17]. However, the underlying mechanism(s) involved in acaricide detoxification and resistance in D. gallinae remain unknown. In addition to active site mutation, the development of acaricide resistance in other pest species may be attributed to the activities of detoxifying enzymes including 
members of the glutathione $S$-transferase (GST), cytochromeP450 (CYP450), and carboxylesterase (CBE) superfamilies and may be multi-factorial in nature [18]. The CYP450 and CBEs function in phase I of detoxification (modification) by catalyzing the addition of polar groups to xenobiotics. Phase II (conjugation) most often involves the GST-catalysed conjugation of reduced glutathione (GSH) to the electrophilic xenobiotic [19]. The glutathione conjugate acts as a flag, targeting the xenobiotic to the membrane transporters, whereupon it is excreted from the cell in phase III of the detoxification process [20].

GSTs belong to a large superfamily of versatile proteins with important functions in detoxification, amino acid catabolism, signal modulation, transportation [21]. Cytosolic and mitochondrial GSTs are classified in several canonical classes (mu, kappa, alpha, pi, theta, sigma, zeta, omega, lambda, beta, tau, delta and epsilon) dependent on the structure of class-specific active site motifs, active site residues and binding affinities $[22,23]$.

GSTs are highly important in the detoxification processes in haematophagous parasites, particularly in the elimination of toxic haemoglobin breakdown products [24-26]. GSTs have also been identified as major allergens in several parasite and pest species (e.g. scabies and house dust mites, parasitic nematodes and cockroaches), that provoke pathophysiology associated with allergy [27-31]. In addition, secreted GSTs have been implicated in immune-evasion of the nitric oxide response in helminths [32]. Given their pivotal role in detoxification, drug resistance and host-parasite interaction, GSTs have been the focus of novel control strategies against a number of ecto- and endoparasites [33-38].

The identification of D. gallinae GSTs (Deg-GSTs) will therefore lead to a deeper understanding of acaricide resistance in this species and may lead to novel targeted control methods. We have previously produced a D. gallinae transcriptomic database [39] and, in the present study, we identify the repertoire of GSTs in D. gallinae by data-mining the database with GST search terms and investigate the interactions of 3 selected Deg-GSTs with acaricides commonly used in the poultry sector to characterise the potential for involvement of the Deg-GSTs in acaricide detoxification.

\section{Methods}

\section{Generation of $D$. gallinae transcriptome dataset}

Mixed stage and gender D. gallinae mites collected by scraping aggregated mites from the enriched-cage system of a commercial egg-laying unit in Scotland, UK. Mites were allowed to migrate away from the detritus and were then snap frozen in liquid nitrogen within $12 \mathrm{~h}$ of collection. Total RNA was purified using Trizol reagent (Life Technologies) as previously described Wright [39]. Roche 454 sequencing was performed (Edinburgh
Genomics, University of Edinburgh) following the manufacturer's protocol [40, 41]. The raw read data were assembled using Newbler (version 2.3) into contig sequences and putative biological functions inferred by homology searching against the NCBI nr database (19 July 2013 release) using BLASTx [42]. Where possible, the $D$. gallinae trancriptomic dataset was further annotated with Gene Ontology (GO) terms, Enzyme Commission (EC) codes and InterProScan (IPS) identities using the Blast2GO pipeline [43-45].

\section{Amplification and sequencing of selected $D$. gallinae glutathione S-transferases (Deg-GSTs)}

The complete coding sequences of selected Deg-GSTs were obtained using rapid amplification of cDNA ends (RACE) using the SMART ${ }^{\mathrm{max}}$ RACE cDNA amplification kit (Clontech) according to the manufacturer's touchdown protocol and where necessary or, where full length sequence was already represented in the assembled contigs, by direct amplification from cDNA by PCR using the Advantage 2 PCR Kit (Clontech). Sequences of cloned cDNAs representing three GSTs, Deg-GST-1, -2 and -3 were determined following cloning in pGEM $^{\circ}$ T-Easy plasmid (Promega). Homology searching of translated $D e g$-GST sequences against the UniProtKB database (release 2013/06) was performed using blastp. Additionally, Signal P v4.0 [46] was used to identify putative signal sequences and ScanProsite [47] to identify functional domains and protein signature sequences in the translated Deg-GST sequences. A selection of proteins with highest homology to the Deg-GSTs were aligned with the translated Deg-GST along with arthropod and eukaryotic GSTs belonging to selected GST classes, using ClustalX v2.0.11 [48]. The constituent proteins were manually truncated at $\mathrm{C}$ and $\mathrm{N}$ terminus by up to 29 amino acids in order to eliminate regions of extensive gapping and poor alignment. The truncated alignment was imported into the TOPALi v2.5 [49] and the Whelan \& Goldman model (WAG) amino acid substitution model was applied to the alignment to calculate distances. A phylogenetic tree was constructed using the MrBayes Bayesian tree method [50] using default parameters. The integrity of each partition or clade (clade credibility) was assessed using posterior probabilities.

\section{Expression, purification and activity of recombinant Deg-GSTs (rDeg-GSTs)}

The complete coding sequences of the Deg-GSTs were subcloned into the pETSUMO champion plasmid vector (Invitrogen, primer sequences and conditions available on request from authors). For recombinant protein expression, purified pETSUMO-Deg-GST plasmid DNAs were transformed into Escherichia coli BL21-CodonPlus ${ }^{\oplus}$ (DE3)-RIL competent cells (Stratagene) and protein 
expression, purification and quantification performed as described previously [51]. Purified $\mathrm{r} D e g$-GSTs were used for electrophoresis on NuPAGE Bis-Tris 4-12 \% gels under reducing conditions and stained with SimplyBlue ${ }^{\mathrm{rm}}$ (Invitrogen). To confirm identity of the $\mathrm{rDeg}$-GSTs, the single visible bands were excised, destained and subjected to reductive alkylation using DTT and iodoacetamide. Gel pieces were digested overnight at $37{ }^{\circ} \mathrm{C}$ in trypsin and digests analysed on an Ultraflex II MALDIToF-ToF mass spectrometer (Bruker Daltonics). The masses obtained were used for database searching with the MASCOT search engine using Swiss-Prot and local databases with a $50 \mathrm{ppm}$ mass tolerance window. Significant matches from the Peptide Mass Fingerprint data were confirmed by MS/MS analysis using the search criteria above and an MS/MS tolerance window of 0.5 Da.

The ability of the purified rDeg-GSTs to catalyse the conjugation of reduced glutathione (GSH) to 1-chloro2,4-dinitrobenzene (CDNB) was assessed and quantified by measuring the increase in absorbance at $340 \mathrm{~nm}$ $\left(\mathrm{A}_{340 \mathrm{~nm}}\right)$ over time as described in Lee et al. [52]. Briefly, $20 \mu \mathrm{l}$ volumes containing defined quantities of the $\mathrm{r} D e g$ GSTs in Tris/ $\mathrm{NaCl}$ buffer $(10 \mathrm{mM}$ Tris, $0.5 \mathrm{M} \mathrm{NaCl}$, $\mathrm{pH}$ 7.4) were placed, in triplicate, into the test wells of a 96-well microtitre plate and the equivalent volume of Tris/ $\mathrm{NaCl}$ buffer into the control wells. $100 \mu \mathrm{l} C D N B$ at serial concentration range of $0 \mathrm{mM}$ to $1.0 \mathrm{mM}$ (in $0.2 \mathrm{mM}$ increments) were added in substrate buffer $(100 \mathrm{mM}$ potassium dihydrogen phosphate, $1 \mathrm{mM}$ EDTA, $\mathrm{pH}$ 6.5; $2 \mathrm{mM}$ GSH), to all wells. The $\mathrm{A}_{340 \mathrm{~nm}}$ was measured immediately and every minute thereafter for 20 min (ELx808IU microplate reader, BIO-TEK instruments, Inc.) while the reaction proceeded in a linear phase. The mean $A_{340 \mathrm{~nm}}$ of the triplicate control wells was subtracted from the mean of the triplicate tests wells at the different CDNB concentrations to adjust for spontaneous hydrolysis of CDNB. The specific activities $(\mu \mathrm{mol} / \mathrm{min} / \mathrm{mg}$ protein) of $\mathrm{rDeg}$ GSTs at the different CDNB concentrations were calculated using the formula:

$$
\frac{(\text { At2-At1 }) \times 1000}{\mathrm{~A} \varepsilon \times(\mathrm{t} 2-\mathrm{t} 1) \times \mathrm{b} \times \mathrm{m}} \times \mathrm{Vtot}
$$

where the At1 and At2 are the mean adjusted $\mathrm{A}_{340 \mathrm{~nm}}$ at the initial and final time points; $\Delta \varepsilon$ the molar extinction coefficient of CDNB $(\Delta \varepsilon=9.6)$. $\mathrm{t} 2-\mathrm{t} 1$ is the time (in minutes) between At2 and At1; b is the path length of the spectrophotometer $(\mathrm{cm}=0.286) ; \mathrm{m}$ is the quantity of $\mathrm{rDeg}$-GST per well (in $\mathrm{mg}$ ) and Vtot is the total volume per well (litres).

\section{Acaricide inhibition assays}

$5 \mathrm{mM}$ stocks of the acaricides: spinosad, permethrin, phoxim and abamectin (PESTANAL ${ }^{\circ}$ analytical standards,
Sigma) and $50 \mathrm{mM}$ 4-nitrobenzyl chloride (NBC) were prepared in ethanol. Acaricide, NBC (+ve control) or ethanol (no treatment control) was placed $(2.4 \mu \mathrm{l})$ into triplicate wells of a 96-well microtitre plate and mixed with $20 \mu \mathrm{r}$ Deg-GST [containing defined quantities of $\mathrm{rDeg}$-GSTs in Tris/NaCl buffer $(10 \mathrm{mM}$ Tris, $0.5 \mathrm{M}$ $\mathrm{NaCl}, \mathrm{pH}$ 7.4)]. The plate was incubated for 10 mins at R.T. with agitation prior to the addition of $100 \mu \mathrm{l}$ per well of $1 \mathrm{mM}$ CDNB substrate in substrate buffer. GST activity was measured as detailed above and adjusted for background absorbance of the different acaricides at $A_{340 \mathrm{~nm}}$. The assay was repeated on 3 separate occasions and the mean enzyme activities analysed using a oneway ANOVA followed by pairwise comparisons using Tukey's post-hoc analysis to assess significance.

\section{Results}

Identification and characterisation of Dermanyssus gallinae GST transcripts

The annotated $D$. gallinae transcriptome containing 13,363 contiguous sequences (contigs) [39] was interrogated using glutathione $S$-transferase keywords and 11 contigs were identified with GST terms. These 11 contigs ranged from 208 to $1030 \mathrm{bp}$ in length and, based on BLASTx descriptions, 6 were putatively identified as mu class, 3 as mitochondrial kappa class, 1 omega and 1 delta class.

The 3 longest contigs with the highest number of constituent sequences and top hit BLASTx e values were selected for further study. The contig forming Deg-GST-1 was 1016 bp in length, comprised of 58 overlapping sequences and had a $48 \%$ identity (over 237 amino acid residues, $\mathrm{E}=8 \mathrm{e}-72$ ) with a putative mu-1 like GST from the highly related predatory mite species Metaseiulus occidentalis (accession number XP_003747409). The 1030 bp contig representing Deg-GST-2 comprised of 33 overlapping sequences and possessed $77 \%$ identity (over 208 amino acid residues, 1.0E-77) with a putative $M$. occidentalis GST isoform C-like GST (accession number XP_003743487). The contig representing Deg-GST -3 was formed from 5 overlapping sequences, had a total length of $399 \mathrm{bp}$ and a $76 \%$ identity (over 66 amino acid residues, $2.0 \mathrm{E}-25$ ) with a putative $M$. occidentalis mu-1 like GST (accession number XP_003742682).

The contigs representing Deg-GST-1 and -2 were both predicted to contain the full coding sequence (CDS) of their respective GSTs (Accession numbers KR337505 and KR337506) and this was confirmed using PCR amplification. The contig representing Deg-GST-3 encoded a partial open reading frame (ORF) and the full length sequence (Accession Number KR337507) was obtained using RACE. The ORF of Deg-GST-1 was 735 bp in length, encoding a 244 amino-acid (aa) protein; the ORF of Deg-GST-2 was 633 bp encoding a 210 aa protein and 
the ORF of Deg-GST-3 was 669 bp encoding a 222 aa protein.

All 3 Deg-GSTs possessed GST N-terminal (IPRO04045) and GST C -terminal (IPR004046 and IPRO10987) motifs as well as N-terminal thioredoxin-like fold (IPRO12336) and GST C-terminal chloride channel (IPRO17933) motifs which are typical of all classes of GSTs. Database scanning identified the four elements of the mu class signature sequence motif (PRINTS: PRO1267) in Deg-GST-3, however these elements were not observed in the other putative mu class GST, Deg-GST-1 (Fig. 1). Likewise, a loop structure characteristic of mu class GSTs was highly conserved in Deg-GST-3 $\left(\mathrm{G}^{34}\right.$ PAGPFD $\left.^{40}\right)$, but poorly conserved in Deg-GST-1. The divergence of Deg-GST-1 protein sequence from typical mu class GST was also apparent from several short sequence insertions that are not present in any of the other mu class aligned sequences, except $M$. occidentalis where these insertions are partially conserved. Despite the apparent divergence of the two putative mu class Deg-GST (-1 and -3 ), both matched the PDBeMotif panther PTHR11571:SF3 subfamily comprised of functional mu class GSTs. The tyrosine amino acid responsible for GST activation in the G-site of mu, phi, alpha and sigma GST classes is conserved in Deg-GST-1 $\left(\mathrm{Tyr}^{9}\right)$ and Deg-GST-3 $\left(\mathrm{Tyr}^{5}\right)$. Activation of the G-site in delta, theta and zeta class GSTs occurs with a serine rather than tyrosine [53] and the $\mathrm{Ser}^{9}$ of Deg-GST-2 is conserved supporting the putative class designation of Deg-GST-2 as theta or delta class.
A phylogenetic analysis of the 3 Deg-GSTs and 50 representative GSTs from vertebrates and invertebrates, encompassing alpha, mu, beta, zeta, omega, delta, epsilon and theta classes is presented in Fig. 2. Both DegGST-1 and Deg-GST-3 partitioned with the mu class GST clade. Within the mu clade, the GSTs grouped into 3 defined subclades, supported by a strong clade credibility $(C C=1.0)$. The first mu subclade contains members of the superorder Acariformes (e.g. Sarcoptes spp. and Psoroptes spp. mites). The second mu subclade partitioned further into 3 clusters containing mammalianand crustacean-derived GSTs, those from the Mesostigmatid mites and the Ixodid tick species. The third subclade also partitioned into 2 clusters containing the Mesostigmatid mites and Ixodida ticks respectively. Both putative mu class Deg-GST s $(-1$ and -3$)$ partitioned mostly closely with the Mesostigmatid mite $M$. occidentalis.

Deg-GST-2 partitioned into a large clade containing delta and epsilon GSTs. The representative epsilon GST species formed a cluster discrete from the delta GSTs which, in turn, formed one cluster containing mainly acarine-derived sequences and one cluster containing exclusively insect-derived sequences. As with the DegGST mu class GSTs, the Deg-GST-2 also partitioned most closely with $M$. occidentalis.

\section{Deg-GST activity}

All three rDeg-GST- SUMO-fusion proteins were successfully expressed in E. coli as soluble recombinant

\begin{tabular}{|c|c|c|}
\hline $\begin{array}{l}\text { from Dermanyssus gallinae. The N-termina } \\
\text { with their two closest BLASTp matches: } N \\
\text { Haemaphysalis longicornis (AAQ74441.1). } \\
\text { shading), the four mu class elements (PRC } \\
\text { Deg-GST-1 and XP_003747409.1 are in bo }\end{array}$ & 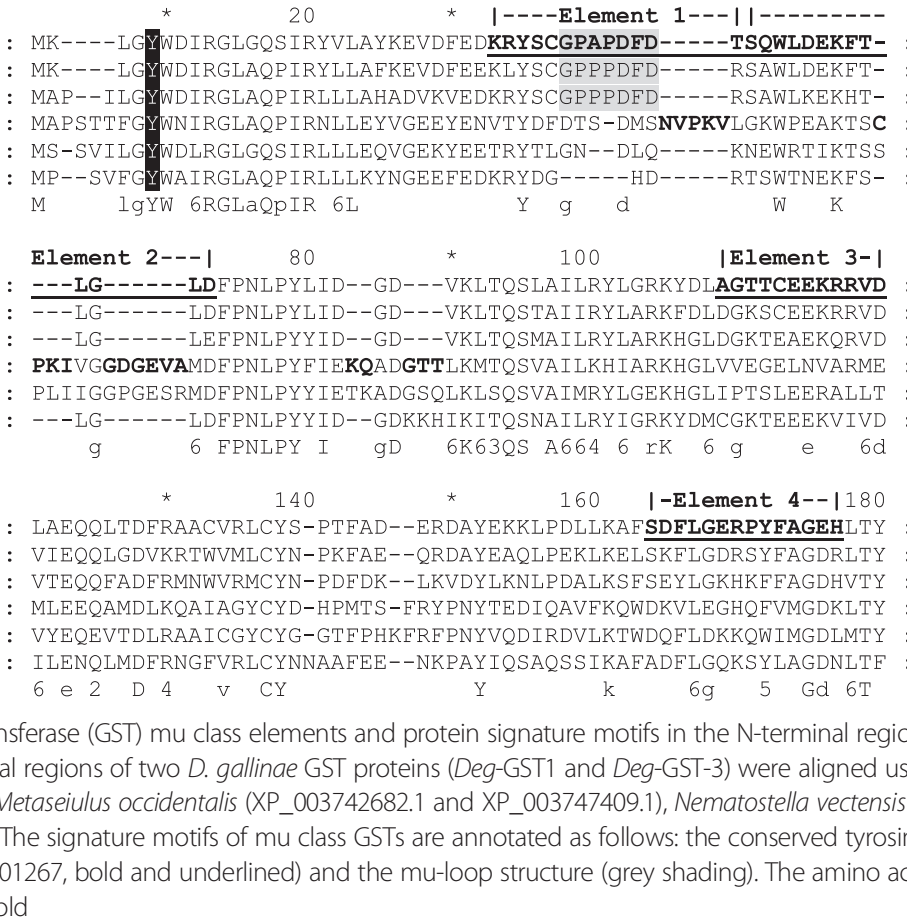 & $\begin{array}{l}\text { ion of two GSTs derived } \\
\text { ssing the ClustalX algorithm } \\
\text { is (XP_001634653.1) and } \\
\text { ine active site residue (black }\end{array}$ \\
\hline
\end{tabular}




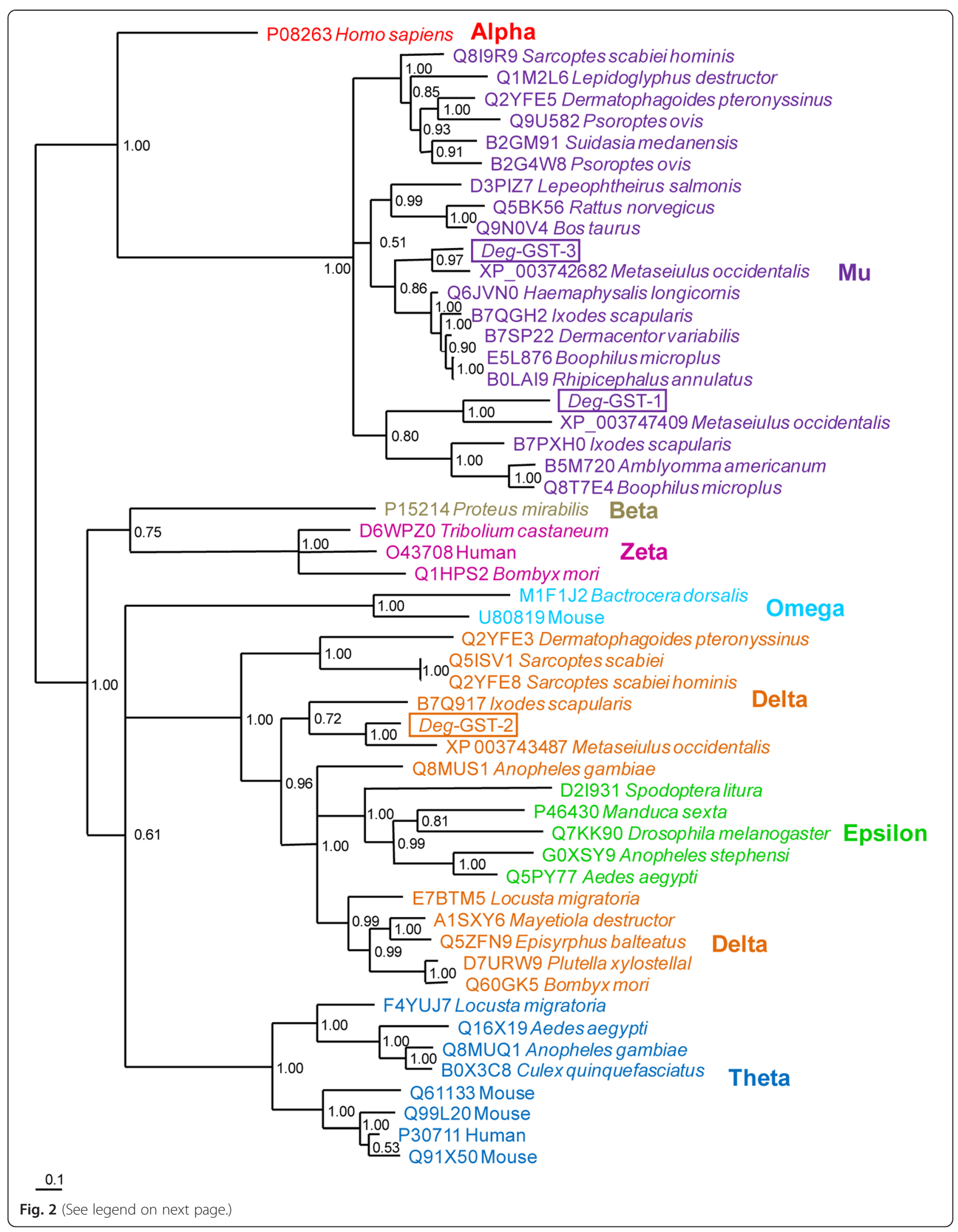


(See figure on previous page.)

Fig. 2 Phylogentic analysis of three glutathione S-transferase (Deg-GST) protein sequences derived from Dermanyssus gallinae. Three Deg-GST protein sequences (Deg-GST -1, -2 and -3) were aligned using the ClustalX algorthim along with a selection of homologous and protypal proteins belonging to the selected classes of GSTs: alpha, mu, beta, zeta, delta, omega epsilon and theta. The constituent proteins were manually truncated at the termini to eliminate regions of poor alignment. The phylogenetic tree was determined using the default parameters of MrBayes Baysian tree method with the Whelan \& Goldman model (WAG) amino acid substitution model. The accession number and species of each protein is presented. The 3 Deg-GSTs are boxed. The clade credibility of each partition was assessed using posterior probabilities and is presented at each node

proteins. Following affinity purification and dialysis to remove imidazole, the rDeg-GSTs were analysed by SDS-PAGE and the inferred molecular masses of the purified proteins were consistent with the calculated masses of the respective fusion proteins (Fig. 3). The identities of the purified rDeg-GST proteins were confirmed using MALDI-ToF mass spectrometry.

All three $\mathrm{rDeg}$-GSTs were enzymically-active molecules capable of catalyzing the conjugation of GSH to CDNB substrate. The activity of each enzyme ( $\mu \mathrm{mol}$ CDNB conjugated with $\mathrm{GSH} / \mathrm{min} / \mathrm{mg}$ GST) was calculated and plotted against CDNB concentration (Fig. 4). A linear relationship was observed between the $\mathrm{r} D e g$ GST and substrate concentrations within this range. rDeg-GST-1 possessed the highest specific activity. Comparison of the activity of the three rDeg-GSTs with CDNB substrate at a concentration of $0.4 \mathrm{mM}$ showed that $\mathrm{r}$ Deg-GST-1 was 18.3 times more active than $\mathrm{rDeg}$ GST-2 and 26.8 times more active than $\mathrm{rDeg}$-GST-3.

\section{Interaction of Deg-GSTs with acaricides}

The ability of several acaricides to interact with the $\mathrm{rDeg}$-GSTs and thus inhibit their ability to catalyze the conjugation of GSH to CDNB was demonstrated using the acaricide inhibition assay (Table 1). The inhibitory effect of acaricide interaction with the rDeg-GSTs was quantified by measuring the decrease in absorbance at $340 \mathrm{~nm}\left(\mathrm{~A}_{340 \mathrm{~nm}}\right)$. The lowest possible concentrations of rDeg-GSTs (given in Table 1), that allowed a constant GST activity over a 20 min period, in the presence of $1 \mathrm{mM} \mathrm{CDNB}$ and $2 \mathrm{mM} \mathrm{GSH}$, were used in the assays in order to maximise the detection of inhibition. The conjugation of reduced glutathione with CDNB by all 3 $\mathrm{rDeg}$-GSTs was significantly reduced [confidence intervals $(\mathrm{CI}) \geq 95 \%$ ] by the presence of NBC, which served as a competitive-inhibition positive control. Phoxim was the only acaricide to significantly reduce $(\mathrm{CI} \geq 90 \%)$ the activity of all $3 \mathrm{rDeg}$-GSTs. Phoxim also produced the greatest reduction in $\mathrm{rDeg}$-GST activity: a reduction of $56 \%$ with rDeg-GST-1. Conversely, spinosad was the only acaricide that had no significant effect on the activity of any of the rDeg-GSTs tested. Permethrin and abamectin also did not have any significant $(\mathrm{CI} \geq 90 \%)$ effect on the activity of rDeg-GST-2 and -3 , but showed a 35 and $17 \%$ reduction in $\mathrm{r}$ Deg-GST-1 activity respectively.

\section{Discussion}

In this study we have identified the repertoire of DegGSTs in a transcriptomic dataset and selected and $2 \mathrm{mu}$ class and one delta class Deg-GST for further characterisation. We have shown that several commonly used poultry acaricides have the potential to affect the function of recombinant versions of the selected Deg-GSTs. Control of Dermanyssus gallinae is becoming increasingly difficult due to the emergence of acaricide resistance coupled with the legislated withdrawal of previously effective acaricides [5], for example, the organophosphate fenitrothion is now banned in the UK [15]. In addition, the application of acaricides, sometime illegally, has resulted in acaricide residues in eggs, meat and feed at concentrations greater than the maximum residue limits in the EU and elsewhere [54-56]. Our understanding of the mechanisms involved in acaricide resistance in D. gallinae are in their infancy, though organophosphate resistance has clearly been linked with increased acetylcholinesterase (AChE) activity [57]. Molecular studies underpinning our fundamental understanding of poultry red mite biology were also lacking until very recently, when large quantities of transcriptomic data were generated $[39,58,59]$. In the transcriptomic dataset interrogated here, the most frequent BLASTx term associated with known detoxification pathways was gluthionine $S$-transferase. Of the 11 contigs with associated GST terms, 6 were putatively ascribed to the cytosolic mu class. The bias towards mu class GSTs in D. gallinae was also evident in the D. gallinae transcriptomic data published by Schichts et al. [59], where of the 32 isotigs identified with associated GST terms, 22 were putatively classified as mu. The high representation of mu class GSTs is in agreement with genomic analyses of another haematophagous parasitic acarine species, Ixodes scapularis, where in silico genome analysis revealed that mu was the predominant GST class followed by delta, epsilon, omega, zeta and kappa [60]. This is in contrast to the insect species, Tribolium castaneum and Anopheles gambiae, which lack the mu class entirely; with the mu class replaced by an expanded delta class [61, 62]. Delta class GSTs were canonically defined as insect-specific [19, 63], however, delta class GSTs have now been described here from $D$. gallinae and previously from several other species of the Acari (e.g. Sarcoptes scabiei, I. scapularis [60, 64] and P. ovis [S Burgess, personal communication]. Epsilon and zeta class Deg-GSTs were not identified in the 


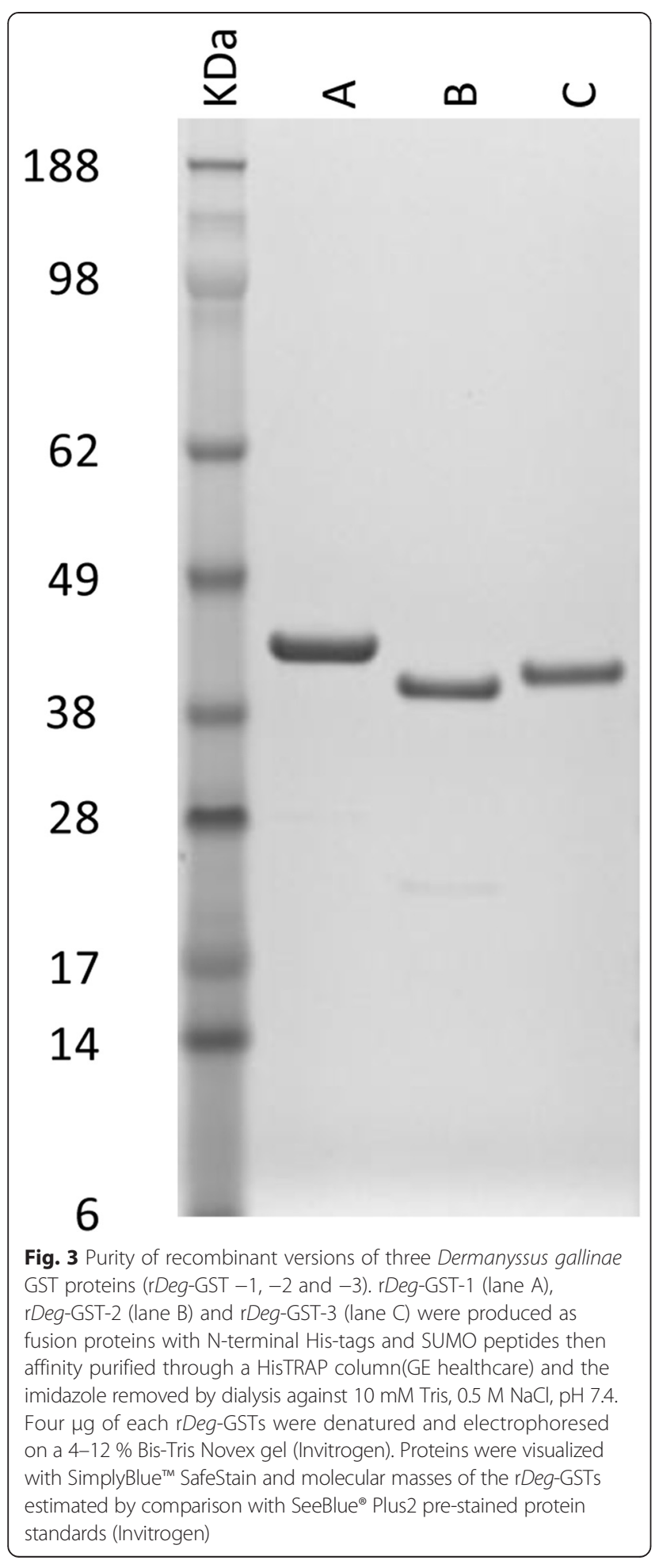

red mite transcriptomic data sets, which was unexpected because they have been identified in other acarine species genomes [60] and epsilon and delta classes are often expanded in other haematophagous arthropods [61]. However, the apparent absence of these classes from the red mite transcriptomic databases may be due to very low levels of gene expression and thus representation in acarine transcriptomic datasets: for example, the $P$. ovis transcriptome (12,160 contig/isotig assembly) has only 2 zeta class GST isotigs and no epsilon class GSTs (personal communication $S$ Burgess). Transcripts representing sigma class GSTs were also absent from the D. gallinae transcriptomic data, which is in agreement with other Acari species e.g. the I. scapularis genome [60] and the P. ovis transcriptome [S. Burgess personal communication].

Phylogenetic analyses of the 3 selected Deg-GSTs consistently placed the Deg-GSTs within the Parasitiformes superorder. Mites belonging to the Acariformes superorder (e.g. the Astigmid mite species P. ovis and S. scabiei) partitioned in a separate clade. In all cases, DegGSTs partitioned most closely with $M$. occidentalis, which is unsurprising as both species belong to the suborder Dermanyssina and M. occidentalis is the most closely taxonomically related species included in the phylogenetic analysis.

All 3 rDeg-GSTs exhibited activity with CDNB; a ubiquitous substrate for all GST classes except theta class [19], however, rDeg-GST-1 was 18 and 26 times more active than rDeg-GST-2 and rDeg-GST-3 respectively. The variation in Deg-GST activity is likely to be the result of underlying primary sequence differences affecting the affinity for CDNB, as even single amino acid substitutions in or near to the active sites of GSTs have been shown to dramatically alter CDNB binding ability [65]. Variation in GST structure arising from allelic variation also impacts on detoxification in vivo and such allelic variation has been associated with altered binding specificity of GSTs to DDT in the mosquito Anopheles gambiae [66]. The generation of alleleic variants provides a mechanism whereby differential pesticide-binding specificities can arise and result in pesticide-tolerant phenotypes. However, no evidence for GST-allelic variation associated with acaricide resistance in Acarid populations has been reported to date.

Here, we tested the ability of a range of commonlyused poultry sector acaricides to interact with $\mathrm{rDeg}$ GSTs and the greatest reduction in GST activity was measured when $\mathrm{rDeg-GST-1}$ activity was inhibited in the presence of the organophosphate compound phoxim. Phoxim was also the only acaricide that produced a statistically significant reduction in activity in all $3 \mathrm{rDeg}$ GSTs indicating that the $3 \mathrm{rDeg}$-GSTs tested here may play a role in phoxim detoxification. Whether the binding between the rDeg-GSTs and phoxim is a functional interaction resulting in a competitive inhibition of GST activity with the CDNB substrate (i.e. phoxim acts as a reducible substrate for GST-mediated GSH conjugation) or whether phoxim is non-competitive inhibitor (i.e. phoxim binds to, and directly inhibits the function of the rDeg-GSTs with conjugation to GSH) is not yet clear. 


\section{A}

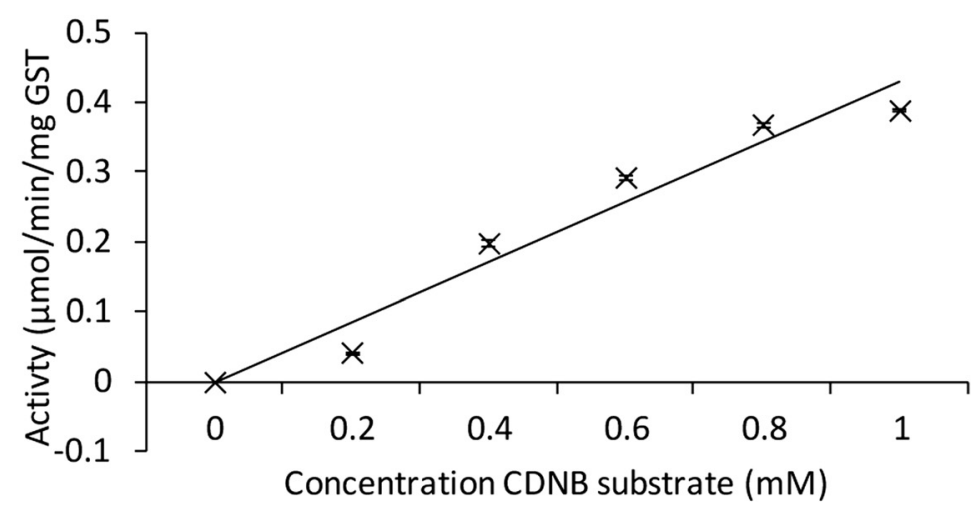

B
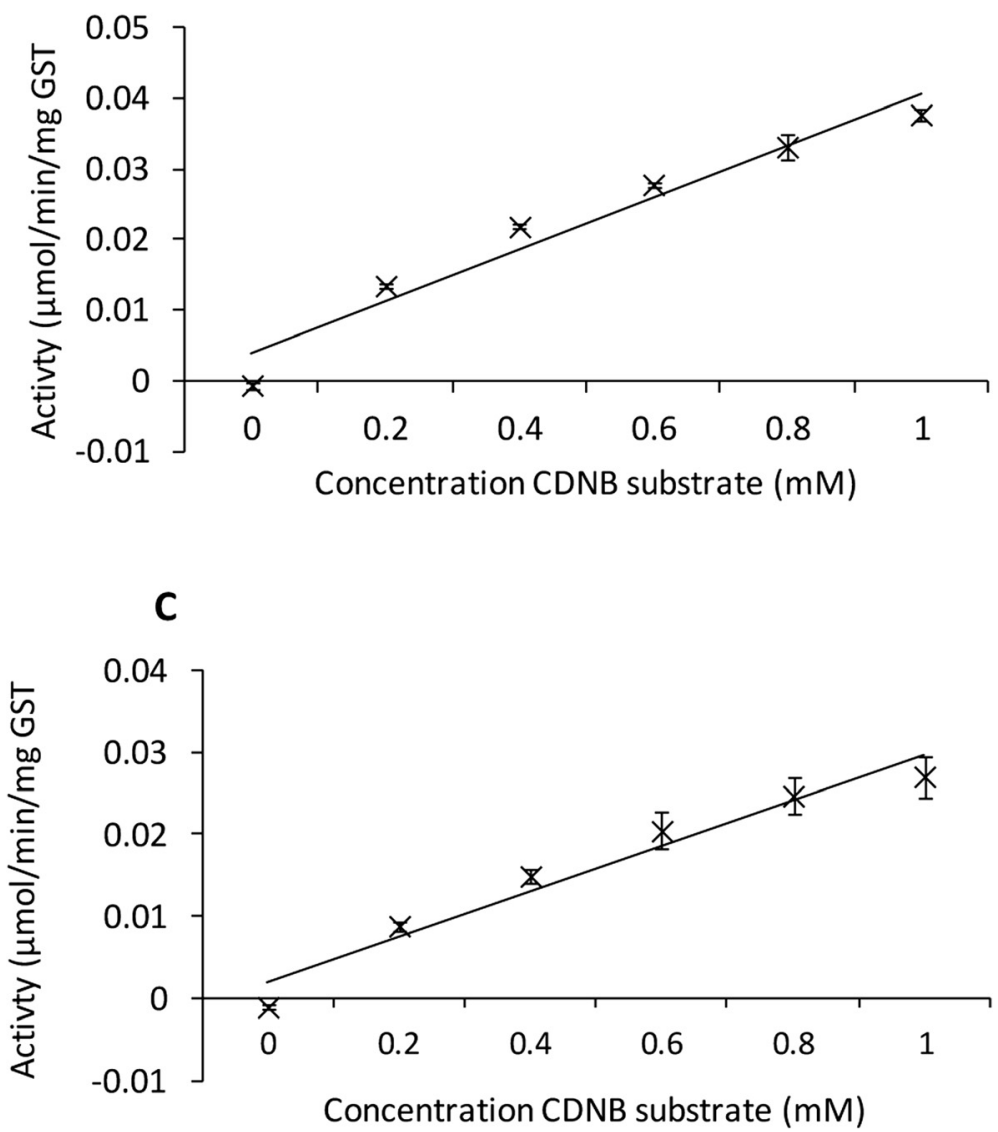

Fig. 4 Enzymatic activity of three recombinant Dermanyssus gallinae GSTs proteins (rDeg-GSTs). The enzymatic activity of the purified rDeg-GST -1, -2 and -3 (panels $\mathbf{a}, \mathbf{b}$ and $\mathbf{c}$ respectively) was determined over a $20 \mathrm{~min}$ period by measuring the increase in absorbance at $340 \mathrm{~nm}\left(A_{340 \mathrm{~nm}}\right)$ resulting from the GST-driven processing of the colorimetric 1-chloro-2,4-dinitrobenzene (CDNB) substrate present at a range of concentrations (0 to $1 \mathrm{mM})$. All assays were performed in triplicate with a constant concentration of $2 \mathrm{mM}$ reduced glutathionine (GSH). The $\mathrm{A}_{340 \mathrm{~nm}}$ was adjusted for spontaneous substrate decay prior to calculating the mean specific activity ( $\mu \mathrm{mol} / \mathrm{min} / \mathrm{mg}$ GST). The mean specific activity at the different CDNB concentrations is shown $( \pm \operatorname{SEM}, n=3)$

Phoxim, and other organophosphates, irreversibly binds to acetylcholinesterase (AChE), thus preventing the AChE-mediated breakdown of acetylcholine (ACh) and the natural cessation of nervous impulses [67]. The resulting overstimulation of the acarine nervous system eventually leads to paralysis and death. In addition to the 
Table 1 The effect of selected acaricides on the ability of rDeg-GSTs to catalyse the conjugation of glutathione to the colorimetric substrate CDNB

\begin{tabular}{lllll}
\hline Treatment & Concentration & \multicolumn{2}{l}{ \% reduction in Deg-GST activity \pm SEM } & \\
& & rDeg-GST-1 $(0.3 \mu \mathrm{g}$ GST/well) & rDeg-GST-2 $(1.5 \mu \mathrm{g}$ GST/well) & rDeg-GST-3 (1.5 $\mu \mathrm{g}$ GST/well) \\
\hline NBC & $1.0 \mathrm{mM}$ & $50.84 \pm 1.4^{\mathrm{b}}$ & $18.1 \pm 1.7^{\mathrm{b}}$ & $33.3 \pm 2.1^{\mathrm{b}}$ \\
Spinosad & $0.1 \mathrm{mM}$ & $-17.46 \pm 3.6$ & $1.7 \pm 5.5$ & $-18.8 \pm 5.7$ \\
Permethrin & $0.1 \mathrm{mM}$ & $35.39 \pm 0.9^{\mathrm{b}}$ & $0.5 \pm 3.0$ & $-0.30 \pm 0.3$ \\
Phoxim & $0.1 \mathrm{mM}$ & $56.38 \pm 3.0^{\mathrm{b}}$ & $14.8 \pm 1.5^{\mathrm{a}}$ & $20.56 \pm 2.5^{\mathrm{a}}$ \\
Abamectin & $0.1 \mathrm{mM}$ & $17.31 \pm 2.1^{\mathrm{a}}$ & $4.3 \pm 4.2$ & $-0.57 \pm 9.6$ \\
No treatment & $\mathrm{NA}$ & $0.0 \pm 0.0$ & $0.0 \pm 0.0$ & $0.0 \pm 0.0$ \\
\hline
\end{tabular}

rDeg-GSTs were incubated together with appropriate defined concentrations of acaricides, 4-nitrobenzyl chloride (NBC) or ethanol diluent (as a no-treatment control) for 10 mins at RT prior to the addition of $1.0 \mathrm{mM}$ CDNB substrate. The final concentrations of acaricides and NBT used in these assay were selected on the basis of their solubility and inhibitory effect in several similar GST inhibition assays [68-70, 85]. The absorbance at $340 \mathrm{~nm}\left(\mathrm{~A}_{340 \mathrm{~nm}}\right)$ was measured immediately (background absorbance of the treatments) and again at 20 mins. The assay was repeated 3 times. The $A_{340 \mathrm{~nm}}$ values at 20 mins were adjusted for background absorbance and the percentage reduction in Deg-GST activity, compared to the no treatment control, calculated and is presented along with \pm SEM. Differences in the activities of each rDeg-GST in the presence or absence of each acaricide were assessed using a one-way ANOVA followed by pairwise comparison using Tukey's post-hoc analysis

${ }^{a}$ Significant $90 \%$ simultaneous confidence intervals using Tukey post-hoc test of ANOVA data

${ }^{\text {b }}$ Significant $95 \%$ simultaneous confidence intervals using Tukey post-hoc test of ANOVA data

interaction of phoxim with Deg-GSTs shown here, several other commercially-available organophosphates have been shown to interact in vitro with GSTs derived from other acarine species [68-70], indicating a potential role for GSTs in the detoxification or inactivation of organophosphate pesticides in the Acari. A five-fold increase in the expression of a GST transcript in the tick Rhipicephalus microplus following exposure to coumaphos [71, 72] and a ten-fold increase in GST activity in organophosphateresistant Tetranychus urticae mite extract [73] adds weight to the argument that over-expression of GSTs may play an important role in organophosphate detoxification and resistance in the Acari.

The inhibitory effect of permethrin on rDeg-GST activity was limited to rDeg-GST-1, where a $35 \%$ decrease in activity was observed in the acaricide inhibition assay. The interaction between GSTs and permethrin and the inhibition of GST function has also been identified in other tick and mite species using similar acaricide inhibition assays [68-70]. In addition, the increase in GST transcription in response to sublethal pyrethroid treatments in several mite species [74-76] and the increased susceptibility of Rhipicephalus sanguineus sensu lato ticks to the effects of permethrin when an abundant mu class GST is inhibited via RNAi [77] indicates an important role of GSTs in the detoxification processes of pyrethroids in the Acari. The exact nature of the interaction of permethrin with GSTs and their role in detoxification remains unclear: the interaction between the insect Tenebrio molitor GST and decamethrin involved direct binding to the enzyme's active site, but did not result in conjugation of the pesticide with GSH, suggesting sequestration of the decamethrin, thereby preventing it's interaction with the target ligand [78]. Mutations in and/or increased expression of the effector molecules of detoxification pathways are often associated with pesticide resistance and pyrethroid resistance mediated by esterase and CYP450 over-expression as well as SNPs in the voltage-sensitive sodium channel have all been documented in the Acari [72, 74, 79-81].

The increase in GST gene expression and GST activity in response to treatment with pyrthroids (as discussed above) suggests that increased GST activity is also likely to be part of a multi-factorial detoxification system involved in pyrethroid resistance in the Acari. There have been numerous reports of pyrethroid resistance in $D$. gallinae $[12,13,15,17]$ and the potential existence of point mutations in GSTs associated with pyrethroid resistance, allowing increased affinity for pesticides, has been explored in other mites, but thus far none have been identified [82]. The mechanism by which GSTs function in pyrethroid resistance may therefore revolve around increased expression of the enzymes rather than alterations in their structure; thus an increase in the transcription of 6 GSTs in the citrus red mite (Panonychus citri) following exposure to a sublethal dose of fenpropathrin [76]. In S. scabiei, an increase in GST activity in permethrin-resistant mites when compared to permethrin-sensitive mites is associated with mite survival following exposure to permethrin $[74,75]$. Furthermore, when pyrethroidresistant scabies mites were treated with the GST inhibitor and acaricide synergist diethyl malate (DEM), the resistant phenotype was abolished and sensitivity to permethrin restored [75].

Spinosad (Elector ${ }^{\circ}$ ) has recently been registered for use in poultry pest control and has shown to be very effective against all motile stages of $D$. gallinae [83, 84]. No significant change in activity of the $3 \mathrm{rDeg}$-GSTs was observed following incubation with spinosad suggesting that these particular Deg-GSTs do not have high affinity 
for this acaricide. Abamectin is a macrocyclic lactonecontaining pesticide, which was approved for commercial D. gallinae control in the UK in 2012 [5]. The effect of abamectin on rDeg-GST activity was limited to $17 \%$ reduction in $\mathrm{rDeg}$-GST-1 activity, and, although spinosad and abamectin do not appear to interact to any great extent with the rDeg-GSTs tested here; this does not preclude the possibility of other Deg-GSTs functioning in the detoxification of these compounds.

\section{Conclusions}

We have shown that mu and delta class GSTs are present in D. gallinae and interact with phoxim, permethrin and abamectin acaricide compounds and they therefore may have important roles in the detoxification of several classes of pesticides. To further characterise the role(s) of Deg-GSTs in detoxification and resistance, it would be constructive to perform in vivo studies with mites quantifying the Deg-GST activity levels and gene expression profiles following exposure to acaricides in mite populations with and without defined acaricide exposure and resistance. Identifying Deg-GSTs involved in the detoxification of acaricides may allow the development of targeted treatment design to disrupt detoxification and hinder the mites' ability to survive acaricide treatment. Selectively targeting and disrupting Deg-GST function could therefore potentially work in synergy with existing acaricides to increase their efficacy and prolong their use in the field by overcoming resistance.

\section{Competing interests}

The authors declare that they have no competing interests.

\begin{abstract}
Authors' contributions
HW prepared RNA for 454 sequencing and annotated the transcriptomic data. $\mathrm{RB}$ and $\mathrm{KB}$ cloned the Deg-GSTs and produced purified recombinant versions. $\mathrm{KB}$ performed the enzymic assays and prepared the draft manuscript. AN, JH and $\mathrm{KB}$ participated in the study design and revision of the manuscript. All authors read and approved the manuscript.
\end{abstract}

\section{Acknowledgements}

The authors gratefully acknowledge funding for this project from the British Egg Marketing Board Trust and from DEFRA (grant number AW 00186). We would like to also acknowledge continued support from Mr. John Campbell, Glenrath Farms Ltd. for his continued enthusiasm and help in our red mite research. We would also like to thank Professor Mark Blaxter (Edinburgh Genomics) for the 454 transcriptome sequencing, The Moredun Proteomics Group for the MALDI-ToF-ToF and Dr Stewart Burgess for the P. ovis transcriptome data.

Received: 5 May 2015 Accepted: 17 June 2015

Published online: 26 June 2015

\section{References}

1. Van Emous R. Wage war against the red mite! Poultry Int. 2005;4:26-33.

2. Sparagano O, Pavlicevic A, Murano T, Camarda A, Sahibi H, Kilpinen O, et al. Prevalence and key figures for the poultry red mite Dermanyssus gallinae infections in poultry farm systems. Exp Appl Acarol. 2009:48:3-10. doi:10.1007/s10493-008-9233-z.

3. Kilpinen O, Roepstorff A, Permin A, Norgaard-Nielsen G, Lawson LG, Simonsen HB. Influence of Dermanyssus gallinae and Ascaridia galli infections on behaviour and health of laying hens (Gallus gallus domesticus). Br Poult Sci. 2005;46:26-34. doi:10.1080/00071660400023839.

4. Mul FM. Control of Poultry Red Mite in layer farms using an automated monitoring device Prevalence and effects of Dermanyssus gallinae. In: van Neikerk GCMT, Meerburg GB, Groot-Keorkamp WGP, editors. XVIIIth World Veterinary Poultry Association Congress Proceedings. 2013; France.

5. Sparagano OA, George DR, Harrington DW, Giangaspero A. Significance and control of the poultry red mite, Dermanyssus gallinae. Annu Rev Entomol. 2014;59:447-66. doi:10.1146/annurev-ento-011613-162101.

6. Chirico J, Eriksson H, Fossum O, Jansson D. The poultry red mite, Dermanyssus gallinae, a potential vector of Erysipelothrix rhusiopathiae causing erysipelas in hens. Med Vet Entomol. 2003;17:232-4.

7. Valiente Moro C, Desloire S, Vernozy-Rozand C, Chauve C, Zenner L. Comparison of the VIDAS system, FTA filter-based PCR and culture on SM ID for detecting Salmonella in Dermanyssus gallinae. Lett Appl Microbiol. 2007;44:431-6. doi:10.1111/j.1472-765X.2007.02119.x.

8. Valiente Moro C, Chauve C, Zenner L. Experimental infection of Salmonella Enteritidis by the poultry red mite, Dermanyssus gallinae. Vet Parasitol. 2007;146:329-36. doi:10.1016/j.vetpar.2007.02.024.

9. Valiente Moro C, De Luna CJ, Tod A, Guy JH, Sparagano OA, Zenner L. The poultry red mite (Dermanyssus gallinae): a potential vector of pathogenic agents. Exp Appl Acarol. 2009;48:93-104. doi:10.1007/s10493-009-9248-0.

10. Brannstrom S, Hansson I, Chirico J. Experimental study on possible transmission of the bacterium Erysipelothrix rhusiopathiae to chickens by the poultry red mite, Dermanyssus gallinae. Exp Appl Acarol. 2010;50:299-307. doi:10.1007/s10493-009-9317-4.

11. Chu TT, Murano T, Uno Y, Usui T, Yamaguchi T. Molecular detection of avian pathogens in poultry red mite (Dermanyssus gallinae) collected in chicken Farms. J Vet Med Sci. 2014;76:1583-7. doi:10.1292/jvms.14-0253.

12. Zeman P, Zelezny J. The susceptibility of the poultry red mite, Dermanyssus gallinae (De Geer, 1778), to some acaricides under laboratory conditions. Exp Appl Acarol. 1985;1:17-22.

13. Beugnet F, Chauve C, Gauthey M, Beert L. Resistance of the red poultry mite to pyrethroids in France. Vet Rec. 1997;140:577-9.

14. Nordenfors $\mathrm{H}$, Hoglund J, Tauson R, Chirico J. Effect of permethrin impregnated plastic strips on Dermanyssus gallinae in loose-housing systems for laying hens. Vet Parasitol. 2001;102:121-31. doi:10.1016/S0304-4017(01)00528-3.

15. Fiddes MD, Le Gresley S, Parsons DG, Epe C, Coles GC, Stafford KA. Prevalence of the poultry red mite (Dermanyssus gallinae) in England. Vet Rec. 2005;157:233-5.

16. Thind BB, Ford HL. Assessment of susceptibility of the poultry red mite Dermanyssus gallinae (Acari: Dermanyssidae) to some acaricides using an adapted filter paper based bioassay. Vet Parasitol. 2007;144:344-8. doi:10.1016/j.vetpar.2006.10.002.

17. Marangi M, Cafiero MA, Capelli G, Camarda A, Sparagano OA, Giangaspero A. Evaluation of the poultry red mite, Dermanyssus gallinae (Acari: Dermanyssidae) susceptibility to some acaricides in field populations from Italy. Exp Appl Acarol. 2009;48:11-8. doi:10.1007/s10493-008-9224-0.

18. Li X, Schuler MA, Berenbaum MR. Molecular mechanisms of metabolic resistance to synthetic and natural xenobiotics. Annu Rev Entomol. 2007:52:231-53. doi:10.1146/annurev.ento.51.110104.151104.

19. Sheehan D, Meade G, Foley VM, Dowd CA. Structure, function and evolution of glutathione transferases: implications for classification of non-mammalian members of an ancient enzyme superfamily. Biochem J. 2001;360:1-16.

20. Cole SP, Deeley RG. Transport of glutathione and glutathione conjugates by MRP1. Trends Pharmacol Sci. 2006;27:438-46. doi:10.1016/j.tips.2006.06.008.

21. Sheerat PJ, Hayes JD. Glutathione S-transferases. In: Ioannides C, editor. Enzyme Systems that Metabolise Drugs and other Xenobiotics. UK: John Wiley and Sons Ltd; 2001. p. 319-51.

22. Dourado DF, Fernandes PA, Ramos MJ. Mammalian cytosolic glutathione transferases. Curr Protein Pept Sci. 2008;9:325-37.

23. Mashiyama ST, Malabanan MM, Akiva E, Bhosle R, Branch MC, Hillerich B, et al. Large-scale determination of sequence, structure, and function relationships in cytosolic glutathione transferases across the biosphere. PLoS Biol. 2014;12:e1001843. doi:10.1371/journal.pbio.1001843.

24. van Rossum AJ, Jefferies JR, Rijsewijk FA, Lacourse EJ, Teesdale-Spittle P, Barrett J, et al. Binding of hematin by a new class of glutathione transferase from the blood-feeding parasitic nematode Haemonchus contortus. Infect Immun. 2004;72:2780-90

25. Perally S, Lacourse EJ, Campbell AM, Brophy PM. Heme transport and detoxification in nematodes: subproteomics evidence of differential role of glutathione transferases. J Proteome Res. 2008;7:4557-65. doi:10.1021/pr800395x. 
26. Toh SQ, Glanfield A, Gobert GN, Jones MK. Heme and blood-feeding parasites: friends or foes? Parasit Vectors. 2010;3:108. doi:10.1186/1756-3305-3-108.

27. Cui YB, Zhou Y, Wang N, Teng FX, Yu LL, Bian YH, et al. Expression, cloning, and IgE-binding of the full-length dust mite allergen Der $\mathrm{f} 8$. Immunol Res. 2014;60:60-8. doi:10.1007/s12026-014-8553-9.

28. O'Neill GM, Donovan GR, Baldo BA. Cloning and characterization of a major allergen of the house dust mite, Dermatophagoides pteronyssinus, homologous with glutathione S-transferase. Biochim Biophys Acta. 1994;1219:521-8. doi:10.1016/0167-4781(94)90080-9.

29. Dougall A, Holt DC, Fischer K, Currie BJ, Kemp DJ, Walton SF. Identification and characterization of Sarcoptes scabiei and Dermatophagoides pteronyssinus glutathione S-transferases: implication as a potential major allergen in crusted scabies. Am J Trop Med Hyg. 2005;73:977-84.

30. Huang CH, Liew LM, Mah KW, Kuo IC, Lee BW, Chua KY. Characterization of glutathione S-transferase from dust mite, Der $\mathrm{p} 8$ and its immunoglobulin $\mathrm{E}$ cross-reactivity with cockroach glutathione S-transferase. Clin Exp Allergy. 2006:36:369-76. doi:10.1111/j.1365-2222.2006.02447.x.

31. Acevedo N, Mohr J, Zakzuk J, Samonig M, Briza P, Erler A, et al. Proteomic and immunochemical characterization of glutathione transferase as a new allergen of the nematode Ascaris lumbricoides. PLoS One. 2013;8:e78353. doi:10.1371/journal.pone.0078353.

32. Cervi L, Rossi G, Masih DT. Potential role for excretory-secretory forms of glutathione-S-transferase (GST) in Fasciola hepatica. Parasitology. 1999;119:627-33.

33. Nisbet AJ, Halliday AM, Parker L, Smith WD, Kenyon F, Knox DP, et al. Psoroptes ovis: identification of vaccine candidates by immunoscreening. Exp Parasitol. 2008;120:194-9. doi:10.1016/j.exppara.2008.06.008.

34. Parizi LF, Utiumi KU, Imamura S, Onuma M, Ohashi K, Masuda A, et al. Cross immunity with Haemaphysalis longicornis glutathione S-transferase reduces an experimental Rhipicephalus (Boophilus) microplus infestation. Exp Parasitol. 2011;127:113-8. doi:10.1016/j.exppara.2010.07.001.

35. Parizi LF, Reck Jr J, Oldiges DP, Guizzo MG, Seixas A, Logullo C, et al. Multi-antigenic vaccine against the cattle tick Rhipicephalus (Boophilus) microplus: a field evaluation. Vaccine. 2012;30:6912-7. doi:10.1016/ j.vaccine.2012.08.078.

36. Seixas A, Oliveira P, Termignoni C, Logullo C, Masuda A, Da Silva Jr VI. Rhipicephalus (Boophilus) microplus embryo proteins as target for tick vaccine. Vet Immunol Immunopathol. 2012;148:149-56. doi:10.1016/j.vetimm.2011.05.011.

37. Goud GN, Deumic V, Gupta R, Brelsford J, Zhan B, Gillespie P, et al. Expression, purification, and molecular analysis of the Necator americanus glutathione S-transferase 1 (Na-GST-1): a production process developed for a lead candidate recombinant hookworm vaccine antigen. Protein Expr Purif. 2012;83:145-51. doi:10.1016/j.pep.2012.03.013.

38. Bourke CD, Nausch N, Rujeni N, Appleby L, Trottein F, Midzi N, et al. Cytokine responses to the anti-schistosome vaccine candidate antigen glutathione-S-transferase vary with host age and are boosted by praziquantel treatment. PLoS Negl Trop Dis. 2014;8:e2846. doi:10.1371/journal.pntd.0002846.

39. Wright HW. Identification of vaccine candidates against the poultry red mite, Dermanyssus gallinae, PhD thesis. Edinburgh: The University of Edinburgh; 2010.

40. Margulies M, Egholm M, Altman WE, Attiya S, Bader JS, Bemben LA, et al. Genome sequencing in microfabricated high-density picolitre reactors. Nature. 2005;437:376-80. doi:10.1038/nature03959.

41. Wheeler DA, Srinivasan M, Egholm M, Shen Y, Chen L, McGuire A, et al. The complete genome of an individual by massively parallel DNA sequencing. Nature. 2008:452:872-6. doi:10.1038/nature06884

42. 42 Altschul SF, Gish W, Miller W, Myers EW, Lipman DJ. Basic local alignment search tool. J Mol Biol. 1990; 215: 403-410. doi:10.1016/S0022-2836(05)80360-2.

43. Conesa A, Gotz S. Blast2GO. A comprehensive suite for functional analysis in plant genomics. Int J Plant Genomics. 2008; 619832. doi:10.1155/2008/619832

44. Conesa A, Gotz S, Garcia-Gomez JM, Terol J, Talon M, Robles M. Blast2GO: a universal tool for annotation, visualization and analysis in functional genomics research. Bioinformatics. 2005;21:3674-6. doi:10.1093/bioinformatics/bti610

45. Gotz S, Garcia-Gomez JM, Terol J, Williams TD, Nagaraj SH, Nueda MJ, et al. High-throughput functional annotation and data mining with the Blast2GO suite. Nucleic Acids Res. 2008:36:3420-35. doi:10.1093/nar/gkn176.

46. Petersen TN, Brunak S, von HG, Nielsen H. SignalP 4.0: discriminating signal peptides from transmembrane regions. Nat Methods. 2011;8:785-6. doi:10.1038/nmeth.1701.

47. de Castro E, Sigrist CJ, Gattiker A, Bulliard V, Langendijk-Genevaux PS,

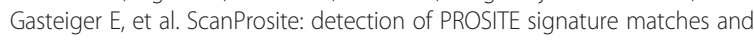

ProRule-associated functional and structural residues in proteins. Nucleic Acids Res. 2006;34:W362-5. doi:10.1093/nar/gkl124.

48. Larkin MA, Blackshields G, Brown NP, Chenna R, McGettigan PA, McWilliam $\mathrm{H}$, et al. Clustal W and Clustal X version 2.0. Bioinformatics. 2007;23:2947-8. doi:10.1093/bioinformatics/btm404.

49. Milne I, Wright F, Rowe G, Marshall DF, Husmeier D, McGuire G. TOPALi: software for automatic identification of recombinant sequences within DNA multiple alignments. Bioinformatics. 2004;20:1806-7. doi:10.1093/bioinformatics/bth155.

50. Ronquist F, Huelsenbeck JP. MrBayes 3: Bayesian phylogenetic inference under mixed models. Bioinformatics. 2003;19:1572-4.

51. Nisbet AJ, Zarlenga DS, Knox DP, Meikle LI, Wildblood LA, Matthews JB. A calcium-activated apyrase from Teladorsagia circumcincta: an excretory/ secretory antigen capable of modulating host immune responses? Parasite Immunol. 2011;33:236-43. doi:10.1111/j.1365-3024.2011.01278.x.

52. Lee AJ, Huntley J, Van den Broek A, Coates D, Isaac RE. Expression and characterisation of a Psoroptes ovis glutathione S-transferase. Vet Parasitol. 2002;105:49-63.

53. Torres-Rivera A, Landa A. Glutathione transferases from parasites: a biochemical view. Acta Trop. 2008;105:99-112. doi:10.1016/j.actatropica.2007.08.005

54. Aulakh RS, Gill JP, Bedi JS, Sharma JK, Joia BS, Ockerman HW. Organochlorine pesticide residues in poultry feed, chicken muscle and eggs at a poultry farm in Punjab, India. J Sci Food Agric. 2006;86:741-4. doi:10.1002/jsfa.2407.

55. Hamscher $\mathrm{G}$, Nau H. Effects of animal housing facilities on residues and contaminants in food. Dtsch Tierarztl Wochenschr. 2003:110:316-9.

56. Marangi M, Morelli V, Pati S, Camarda A, Cafiero MA, Giangaspero A. Acaricide residues in laying hens naturally infested by red mite Dermanyssus gallinae. PLoS One. 2012;7, e31795. doi:10.1371/journal.pone.0031795.

57. Roy L, Chauve C, Delaporte J, Inizan G, Buronfosse T. Exploration of the susceptibility of AChE from the poultry red mite Dermanyssus gallinae (Acari: Mesostigmata) to organophosphates in field isolates from France. Exp Appl Acarol. 2009;48:19-30. doi:10.1007/s10493-009-9249-z

58. Schicht S, Qi W, Poveda L, Strube C. The predicted secretome and transmembranome of the poultry red mite Dermanyssus gallinae. Parasit Vectors. 2013;6:259. doi:10.1186/1756-3305-6-259

59. Schicht S, Qi W, Poveda L, Strube C. Whole transcriptome analysis of the poultry red mite Dermanyssus gallinae (De Geer, 1778). Parasitology. 2014;141:336-46. doi:10.1017/S0031182013001467.

60. Niranjan Reddy BP, Prasad GB, Raghavendra K. In silico characterization and comparative genomic analysis of the Culex quinquefasciatus glutathione S-transferase (GST) supergene family. Parasitol Res. 2011;109:1165-77. doi:10.1007/s00436-011-2364-X.

61. Ding Y, Ortelli F, Rossiter LC, Hemingway J, Ranson H. The Anopheles gambiae glutathione transferase supergene family: annotation, phylogeny and expression profiles. BMC Genomics. 2003:4:35. doi:10.1186/1471-2164-4-35.

62. Shi H, Pei L, Gu S, Zhu S, Wang Y, Zhang Y, et al. Glutathione S-transferase (GST) genes in the red flour beetle, Tribolium castaneum, and comparative analysis with five additional insects. Genomics. 2012;100:327-35. doi:10.1016/j.ygeno.2012.07.010.

63. Board PG, Baker RT, Chelvanayagam G, Jermiin LS. Zeta, a novel class of glutathione transferases in a range of species from plants to humans. Biochem J. 1997;328:929-35.

64. Pettersson EU, Ljunggren EL, Morrison DA, Mattsson JG. Functional analysis and localisation of a delta-class glutathione S-transferase from Sarcoptes scabiei. Int J Parasitol. 2005:35:39-48. doi:10.1016/j.ijpara.2004.09.006.

65. Hansson LO, Widersten M, Mannervik B. An approach to optimizing the active site in a glutathione transferase by evolution in vitro. Biochem $\mathrm{J}$. 1999:344:93-100.

66. Ortelli F, Rossiter LC, Vontas J, Ranson H, Hemingway J. Heterologous expression of four glutathione transferase genes genetically linked to a major insecticide-resistance locus from the malaria vector Anopheles gambiae. Biochem J. 2003;373:957-63. doi:10.1042/BJ20030169.

67. Casida JE, Durkin KA. Anticholinesterase insecticide retrospective. Chem Biol Interact. 2013;203:221-5. doi:10.1016/j.cbi.2012.08.002

68. Da Silva Jr VI, Torino LT, Michelon A, Sanchez Ferreira CA, Joaquim de Freitas DR, Termignoni $C$, et al. Effect of acaricides on the activity of a Boophilus microplus glutathione S-transferase. Vet Parasitol. 2004;119:237-45.

69. da Silva Vaz Jr I, Imamura S, Ohashi K, Onuma M. Cloning, expression and partial characterization of a Haemaphysalis longicornis and a Rhipicephalus appendiculatus glutathione S-transferase. Insect Mol Biol. 2004;13:329-35. doi:10.1111/j.0962-1075.2004.00493.x. 
70. Molin EU, Mattsson JG. Effect of acaricides on the activity of glutathione transferases from the parasitic mite Sarcoptes scabiei. Parasitology. 2008;135:115-23. doi:10.1017/S0031182007003472.

71. Saldivar L, Guerrero FD, Miller RJ, Bendele KG, Gondro C, Brayton KA Microarray analysis of acaricide-inducible gene expression in the southern cattle tick, Rhipicephalus (Boophilus) microplus. Insect Mol Biol. 2008;17:597-606. doi:10.1111/j.1365-2583.2008.00831.x.

72. Guerrero FD, Lovis L, Martins JR. Acaricide resistance mechanisms in Rhipicephalus (Boophilus) microplus. Rev Bras Parasitol Vet. 2012;21:1-6.

73. Nauen R, Stumpf N. Fluorometric microplate assay to measure glutathione $S$-transferase activity in insects and mites using monochlorobimane. Anal Biochem. 2002;303:194-8. doi:10.1006/abio.2002.5578.

74. Pasay C, Arlian L, Morgan M, Gunning R, Rossiter L, Holt D, et al. The effect of insecticide synergists on the response of scabies mites to pyrethroid acaricides. PLoS Negl Trop Dis. 2009;3:e354. doi:10.1371/journal.pntd.0000354.

75. Mounsey KE, Pasay CJ, Arlian LG, Morgan MS, Holt DC, Currie BJ, et al. Increased transcription of Glutathione S-transferases in acaricide exposed scabies mites. Parasit Vectors. 2010;3:43. doi:10.1186/1756-3305-3-43.

76. Liao CY, Zhang K, Niu JZ, Ding TB, Zhong R, Xia WK, et al. Identification and characterization of seven glutathione $S$-transferase genes from citrus red mite, Panonychus citri (McGregor). Int J Mol Sci. 2013;14:24255-70. doi:10.3390/ijms141224255.

77. Duscher GG, Galindo RC, Tichy A, Hummel K, Kocan KM, de la Fuente J. Glutathione S-transferase affects permethrin detoxification in the brown dog tick, Rhipicephalus sanguineus. Ticks Tick Borne Dis. 2014;5:225-33. doi:10.1016/j.ttbdis.2013.11.006.

78. Kostaropoulos I, Papadopoulos Al, Metaxakis A, Boukouvala E, Papadopoulou-Mourkidou E. Glutathione S-transferase in the defence against pyrethroids in insects. Insect Biochem Mol Biol. 2001;31:313-9.

79. Pasay C, Arlian L, Morgan M, Vyszenski-Moher D, Rose A, Holt D, et al. High-resolution melt analysis for the detection of a mutation associated with permethrin resistance in a population of scabies mites. Med Vet Entomol. 2008;22:82-8. doi:10.1111/j.1365-2915.2008.00716.x.

80. Van LT, Vontas J, Tsagkarakou A, Dermauw W, Tirry L. Acaricide resistance mechanisms in the two-spotted spider mite Tetranychus urticae and other important Acari: a review. Insect Biochem Mol Biol. 2010;40:563-72. doi:10.1016/j.ibmb.2010.05.008.

81. Stone NE, Olafson PU, Davey RB, Buckmeier G, Bodine D, Sidak-Loftis LC, et al. Multiple mutations in the para-sodium channel gene are associated with pyrethroid resistance in Rhipicephalus microplus from the United States and Mexico. Parasit Vectors. 2014;7:456. doi:10.1186/s13071-014-0456-z.

82. Shen GM, Shi L, Xu ZF, He L. Inducible Expression of Mu-Class Glutathione S-Transferases Is Associated with Fenpropathrin Resistance in Tetranychus cinnabarinus. Int J Mol Sci. 2014;15:22626-41. doi:10.3390/ijms151222626.

83. George DR, Shiel RS, Appleby WG, Knox A, Guy JH. In vitro and in vivo acaricidal activity and residual toxicity of spinosad to the poultry red mite, Dermanyssus gallinae. Vet Parasitol. 2010;173:307-16. doi:10.1016/j.vetpar.2010.06.035.

84. Liebisch G, Hack R, Smid G. Efficacy of spinosad against the poultry red mite, Dermanyssus gallainae (Mesostigmata: Dermanyssidae), in laboratory and field trials. In: Moraes GJ, Proctor H, editors. Zoosymposia 6. Auckland: Magnolia Press; 2011. p. 282-7.

85. Udomsinprasert R, Pongjaroenkit S, Wongsantichon J, Oakley AJ, Prapanthadara $L A$, Wilce $M C$, et al. Identification, characterization and structure of a new Delta class glutathione transferase isoenzyme. Biochem J. 2005;388:763-71.

\section{Submit your next manuscript to BioMed Central and take full advantage of:}

- Convenient online submission

- Thorough peer review

- No space constraints or color figure charges

- Immediate publication on acceptance

- Inclusion in PubMed, CAS, Scopus and Google Scholar

- Research which is freely available for redistribution

Submit your manuscript at www.biomedcentral.com/submit 